\title{
Safety Improvement of Nuclear Power Reactor Using Soft Computing Techniques
}

\author{
M.H.Syed Mohamed Muzzammil ${ }^{1}$ and E.A.Mohamed $\mathrm{Ali}^{2}$ \\ Department of Electronics and Communication Engineering National College of Engineering, Tirunelveli, TN, \\ India
}

\begin{abstract}
Nowadays, Nuclear Reactors (NR) are large in scale and complex, they are expected to be operated with high levels of reliability and safety. Hence to increase plant safety, to achieve, maintain system stability and assure satisfactory in order to meet the increasing demands for automated system and to detect and diagnose system failures and malfunctions. When a plant malfunction occurs, a great data influx is occurred. This paper proposes a support system based on neuro fuzzy approach conjunction with Genetic Algorithm that assist alarming and diagnosis system. Throughout this framework Neurofuzzy fault diagnosis system is employed to diagnosis the fault of nuclear reactors. Hence to overcome weak points of both neuro learning and linguistic based approaches by which the integrated system will inherit the strength of both approaches and to optimize the Neurofuzzy outcomes using Genetic Algorithm resulting to show the efficiency is obtained by GA is greater and the inaccurate information of the alarming system also compared with Neurofuzzy diagnosis system.
\end{abstract}

Index Terms- Nuclear Reactors (NR), Artificial Neural Network (ANN), Neurofuzzy, fault diagnosis, Genetic Algorithm (GA)

\section{Introduction}

As the systems become more complex and they need to operate with minimum malfunctioning or breakdown time, reliable automated fault detection and diagnosis system are increasing rapidly. A fault diagnosis system is a kind of operator support system that reduces human mistakes, and to ease the workload of operators by quickly suggesting likely faults based on the highest probability of their occurrence.

A typical NR may have around 2,000 alarms in the Main Control Room (MCR) in addition to the display of analog data. During plant transients, hundreds of alarms may be activated in a short time. In the first few minutes after an accident occurrence, operators in a MCR must perform highly mentally work loaded activities. The operators may be overworked and disorders may result. Information overload and stress may severely affect operators' decision making ability. In such situations, a fault diagnosis system will be very helpful to enhance operators' decision making ability and reduce their workload [1,2].

In the field of artificial intelligence, neural network is the most common used method, the nuclear power plant (NPP) is partitioned to small separate fault diagnosis systems to decrease the quantity of input/output data and make the best processing data that does not confuse neural network with all data of the NPP in same program, and then the outputs of all programs will be collected in a global fault diagnosis program $[3,4,5]$. With the weak feature such as Behavior due to unknown input pattern signals, and to overcome the weak feature a fuzzy logic stage prior to the neural network stage is added. Fuzzy logic incorporates the human-like reasoning style of fuzzy systems through the use of fuzzy sets and a linguistic model consisting of a set of IF-THEN fuzzy rules $[6,7]$.

A hybrid intelligent system results by combining the learning and connectionist structure of neural networks with the human-like reasoning style of fuzzy systems. It is the combinations of artificial neural networks and fuzzy logic. Neurofuzzy hybridization is widely termed as Neurofuzzy system (NFS) [8]. And by using Genetic Algorithm the diagnosis outputs were optimized and this will give a clear view for operators on making the decision. Neural networks and Genetic Algorithms are two techniques for learning and optimization, each with its own strengths and weaknesses. Even though they generally evolved along separate paths. However, recently there have been attempts to combine the two technologies. The genetic algorithms implemented in the toolbox let to solve optimization problems with nonlinear, linear, and bound constraints. The genetic algorithm improves the chances of finding a global solution, due to its random nature. [9-11].

In this paper, the reactor passive safety for NPP and reactor's safety based on Artificial Intelligence and Genetic Algorithm have been introduced. The study of applying Neurofuzzy diagnosis system (NFDS) on the recognition of multiple alarms in NPRs has been introduced.

\section{Problem Formulation}

The diagnosis of faults is approached from a pattern matching perspective in that an input pattern is constructed from multiple alarm symptoms and that symptom pattern is matched to an appropriate output pattern 
that corresponds to fault occurred for accidents diagnosis of the nuclear reactor's data The system using Genetic algorithms (GAs) is designed to optimize the diagnosis results.

\section{Safety Factors Of Npp}

NPPs are complex and large in scale; they have 17 critical Points. Fault diagnosis can be carried out for each point of them. Some essential recommendations have been proposed including the passive safety of nuclear reactors. Moreover, intensive research on AI techniques for present nuclear reactors is presented. The following attributes are essential factors for future reactor designs [12, 13]:

- The reactor should be naturally or passively safe. This means that the reactor should be "inherently" safe and not in need for external safety. In other words, the plant is placed in the most vulnerable condition; operators can withdraw all control rods and simultaneously stop all coolant flow, without any adverse impact.

- The transparency of the safety of the plant must be obvious to both the public and the regulators. The design must support risk-informed regulation (the safety must be demonstrable).

- The plant should be simple to operate, upgrade and maintain for limited staff with less technical expertise.

- The plant design must support short construction time to reduce the cost with the eventual decommissioning in mind. Sizing and design of systems to facilitate rapid disassembly, ease of decontamination, and ease of disposal should be performed.

- Online capability to refuel and perform maintenance.

- The system should ensure minimal environmental impact.

\section{Proposed Methodology}

A proposed technique is applied on detailed fault diagnosis of a central critical node. MATLAB toolboxes are used to implement both Neurofuzzy and genetic algorithm techniques. Throughout this framework, the alarm and fault patterns of Kori II reactor have been employed. The first stage of the proposed technique is multi-layer neural network. That results of training and testing. The second stage is a fuzzy system where the rules are applied to check whether the input pattern is known; else to make two possibilities for unknown alarm pattern of this proposed technique. The third stage is Genetic Algorithm the results of applying initial population and population size to get the optimized weight and bias values of the Neurofuzzy system have been shown in details. Such results ensured that proposed optimized value of NFDS can be used on any NPP.

\section{A. Neuro Fuzzy Approach}

In diagnostic applications, faults are characterized by their symptoms, which can evolve with time, performing a trajectory in the observed variable space. The overall NPR has tight alarming and fault diagnosis system. This system has multi-level alarm and fault diagnosis techniques. Every part of the plant has its own diagnosis system. The overall plant has a global alarming and fault diagnosis system, which links all individual subsystems. As the control system of the plant can be tested by NFDS to define the fault if found, also all parts of the plant can be tested by a pattern recognition NFDS techniques [8].

\section{B. Fault Diagnosis of Kori II Cooling Pump}

This subsection introduces more details on Neurofuzzy fault diagnosis in a certain node, it is the cooling pump. Reactor cooling pump (RCP) is considered one of the most important parts of the NPR ( 1 of 17 critical points). This critical point has 12 alarming signals (a1, a2, a3, ..., a12) and 9 possibility of faults (f1, f2, f3, ... f9). The definition of the faults and their corresponding alarms are tabulated in Table 1.a, b.

Neural pattern recognition tool in MATLAB platform is used to create this neural network; it consists of 12 input nodes, 10 hidden nodes, and 9 output nodes as shown in Fig. 1. Different designs with different number of hidden nodes have been proposed but give great errors that ensured that using 10 hidden nodes give more accurate design with small accepted error. As the error back propagation training algorithm (EBPTA) is running, weights of the NFDS are changing till the allowed RMS error reaches its recommended learning value, and thus learning stops. Neural network training has been achieved using 41 known alarm patterns as shown in Table 3 .

Then by using these weights of the NFDS, the diagnosis of any fault caused by any other alarm patterns can be achieved. The flow chart of the NFDS proposed is shown in Fig. 2, as it consists of two major stages: the first one is the fuzzy system and the second one is the neural network [2].

\section{Testing of Neural Network training Set}

The outputs comparison of both reference training patterns and neural network outputs showed that they are nearly typical as the percentage error is less than $1 \%$. These results are achieved when the network has been trained using 41 known cases as given data. Therefore, the network is well trained and it can easily detect any possible system faults. 


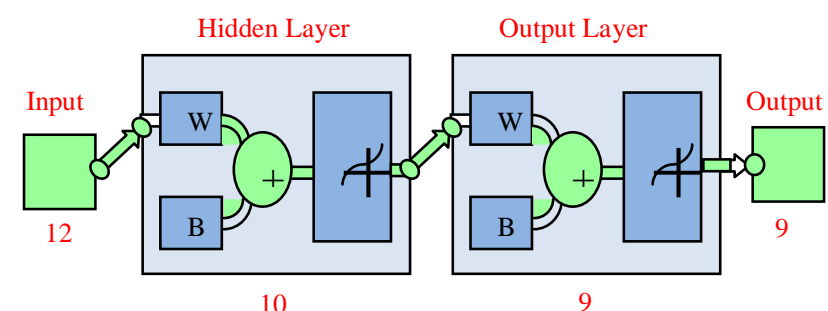

Fig.1. Neural network using neural pattern recognition tool

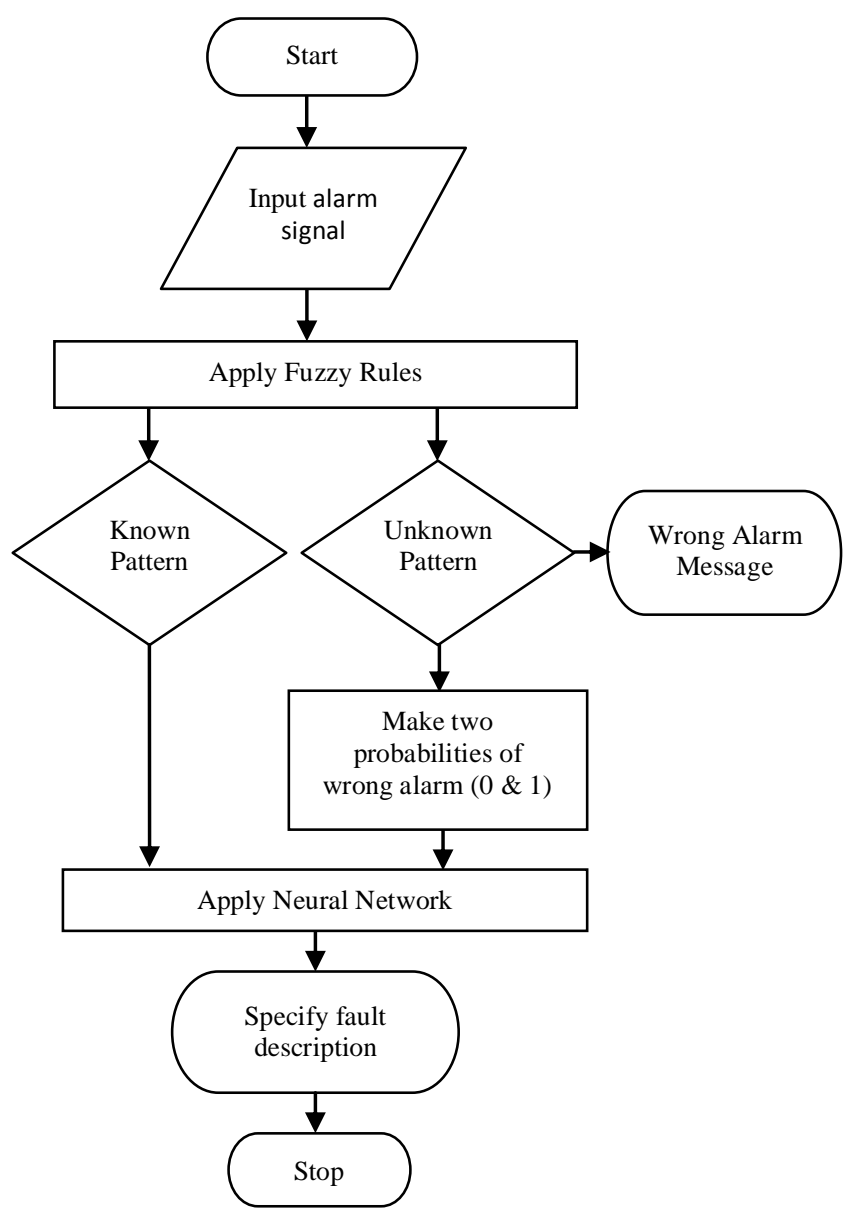

Fig. 2: flow chart of proposed NFDS

\begin{tabular}{|c|l|}
\hline Alarm signal & \multicolumn{1}{|c|}{ Description } \\
\hline $\mathrm{a} 1$ & Seal injection filter differential pressure high \\
\hline $\mathrm{a} 2$ & Charging pump flow low \\
\hline $\mathrm{a} 3$ & Seal injection flow low \\
\hline $\mathrm{a} 4$ & No. 1 Seal differential pressure low \\
\hline $\mathrm{a} 5$ & No. 1 Seal leak off flow low \\
\hline $\mathrm{a} 6$ & Standpipe level low \\
\hline $\mathrm{a} 7$ & Standpipe level high \\
\hline $\mathrm{a} 8$ & No. 1 Seal leak off flow high \\
\hline $\mathrm{a} 9$ & Thermal barrier flow low \\
\hline $\mathrm{a} 10$ & Thermal barrier temperature high \\
\hline $\mathrm{a} 11$ & Bearing flow low \\
\hline $\mathrm{a} 12$ & Bearing temperature high \\
\hline
\end{tabular}

Table (1.a) Alarming signals definitions 


\begin{tabular}{|c|l|}
\hline Fault signal & \multicolumn{1}{|c|}{ Description } \\
\hline f1 & Seal injection filter blockage \\
\hline f2 & Charging pump failure \\
\hline f3 & Seal injection water high temperature \\
\hline f4 & Reactor coolant system pressure less than 400 psig \\
\hline f5 & No. 1 Seal damaged \\
\hline f6 & Volume control tank back pressure high \\
\hline f7 & No. 2 Seal failure \\
\hline f8 & Insufficient component cooling water flow to RCP \\
\hline f9 & Motor Bearing damaged \\
\hline
\end{tabular}

D. Testing of NFDS

Table (1.b) Fault signals definitions

\begin{tabular}{|c|c|c|c|c|c|}
\hline Tested Cases & \multicolumn{2}{|c|}{$\mathrm{i} / \mathrm{p}$ signals } & \multicolumn{2}{|c|}{$\mathrm{o} / \mathrm{p}$ signals } & Notes \\
\hline $\begin{array}{l}\text { Testing with } \\
\text { No alarm } \\
\text { signals }\end{array}$ & & & & & $\begin{array}{l}\begin{array}{c}\text { No alarm } \\
\text { case }\end{array} \\
\text { No fault }\end{array}$ \\
\hline $\begin{array}{l}\text { Testing with } \\
\text { one of the } \\
\text { training } \\
\text { cases }\end{array}$ & & & & & $\begin{array}{l}\text { Case no } 1 \\
\text { The result is } \\
\text { Fault type } \\
\text { (f1) }\end{array}$ \\
\hline $\begin{array}{l}\text { Testing with } \\
\text { two } \\
\text { combined } \\
\text { cases }\end{array}$ & & & & & $\begin{array}{c}\text { Case no } 12 \\
\text { combined } \\
\text { with case } \\
\text { no.13 } \\
\text { The result is } \\
\text { Fault } \\
\text { type(f1) \& } \\
\text { (f2) }\end{array}$ \\
\hline $\begin{array}{l}\text { Testing the } \\
\text { fuzzy } \\
\text { condition } \\
\text { If } 0.2<a<0.8\end{array}$ & & & $\begin{array}{l}0 \\
1 \\
1 \\
5000 \\
0 \\
0 \\
0 \\
0 \\
0 \\
0 \\
0 \\
0\end{array}$ & & $\begin{array}{c}\text { One case } \\
\text { with wrong } \\
\text { alarm signal }\end{array}$ \\
\hline Then "a" & $\mathrm{i} / \mathrm{p} 1$ & $\mathrm{i} / \mathrm{p} 2$ & $\mathrm{o} / \mathrm{p} 1$ & $\mathrm{o} / \mathrm{p} 2$ & $\begin{array}{l}\text { Fault type } \\
\text { (f2) in these }\end{array}$ \\
\hline either 0 or 1 & $\begin{array}{c}0 \\
1 \\
1 \\
0.00 \\
0 \\
0 \\
0 \\
0 \\
\end{array}$ & $\begin{array}{c}0 \\
1 \\
1 \\
1.00 \\
0 \\
0 \\
0 \\
0 \\
\end{array}$ & $\begin{array}{l}0.0000 \\
0.9996 \\
0.0000 \\
0.0000 \\
0.0000 \\
0.0008 \\
0.0001 \\
0.0000 \\
\end{array}$ & $\begin{array}{l}0.0000 \\
0.9996 \\
0.0000 \\
0.0000 \\
0.0000 \\
0.0009 \\
0.0001 \\
0.0000 \\
\end{array}$ & $\begin{array}{l}\text { two results } \\
\text { with alarm } \\
\text { message } \\
\text { "alarm } \\
\text { signal is not } \\
\text { correct" }\end{array}$ \\
\hline
\end{tabular}




\begin{tabular}{|l|l|l|l|l|l|}
\hline & 0 & 0 & 0.0002 & 0.0002 & \\
& 0 & 0 & & & \\
0 & 0 & & & \\
& 0 & 0 & & & \\
\hline
\end{tabular}

Table 2: Testing of the NFDS

Testing NFDS is achieved with no alarm cases, some training cases, combined cases (two known cases are combined in one case) and wrong alarm cases (alarm signals lie between 0.2 and 0.8 ). Because of the limited space of the paper, some of testing results are illustrated in Table 2. As obviously shown, proposed NFDS accurately detects and specifies all fault cases.

\begin{tabular}{|c|c|c|c|c|c|c|c|c|c|c|c|c|}
\hline Case no. & $\mathrm{a} 1$ & $\mathrm{a} 2$ & $\mathrm{a} 3$ & $\mathrm{a} 4$ & a5 & $\mathrm{a} 6$ & a7 & a8 & a9 & a10 & a11 & a12 \\
\hline 1 & 1 & 0 & 0 & 0 & 0 & 0 & 0 & 0 & 0 & 0 & 0 & 0 \\
\hline 2 & 1 & 0 & 1 & 0 & 0 & 0 & 0 & 0 & 0 & 0 & 0 & 0 \\
\hline 3 & 1 & 0 & 1 & 1 & 0 & 0 & 0 & 0 & 0 & 0 & 0 & 0 \\
\hline 4 & 1 & 0 & 1 & 0 & 1 & 0 & 0 & 0 & 0 & 0 & 0 & 0 \\
\hline 5 & 1 & 0 & 1 & 0 & 0 & 0 & 0 & 0 & 0 & 1 & 0 & 0 \\
\hline 6 & 1 & 0 & 1 & 1 & 1 & 0 & 0 & 0 & 0 & 0 & 0 & 0 \\
\hline 7 & 1 & 0 & 1 & 0 & 1 & 1 & 0 & 0 & 0 & 1 & 0 & 0 \\
\hline 8 & 1 & 0 & 1 & 0 & 1 & 0 & 0 & 0 & 0 & 1 & 0 & 0 \\
\hline 9 & 1 & 0 & 1 & 1 & 0 & 0 & 0 & 0 & 0 & 1 & 0 & 0 \\
\hline 10 & 1 & 0 & 1 & 0 & 1 & 1 & 1 & 0 & 0 & 0 & 0 & 0 \\
\hline 11 & 1 & 0 & 1 & 0 & 1 & 1 & 0 & 1 & 0 & 0 & 0 & 0 \\
\hline 12 & 1 & 0 & 1 & 1 & 1 & 0 & 0 & 0 & 0 & 1 & 0 & 0 \\
\hline 13 & 0 & 1 & 0 & 0 & 0 & 0 & 0 & 0 & 0 & 0 & 0 & 0 \\
\hline 14 & 0 & 1 & 1 & 0 & 0 & 0 & 0 & 0 & 0 & 0 & 0 & 0 \\
\hline 15 & 0 & 1 & 1 & 1 & 0 & 0 & 0 & 0 & 0 & 0 & 0 & 0 \\
\hline 16 & 0 & 1 & 1 & 0 & 1 & 0 & 0 & 0 & 0 & 0 & 0 & 0 \\
\hline 17 & 0 & 1 & 1 & 0 & 0 & 0 & 0 & 0 & 0 & 1 & 0 & 0 \\
\hline 18 & 0 & 1 & 1 & 1 & 1 & 0 & 0 & 0 & 0 & 0 & 0 & 0 \\
\hline 19 & 0 & 1 & 1 & 0 & 1 & 1 & 0 & 0 & 0 & 1 & 0 & 0 \\
\hline 20 & 0 & 1 & 1 & 0 & 1 & 0 & 0 & 0 & 0 & 1 & 0 & 0 \\
\hline 21 & 0 & 1 & 1 & 1 & 0 & 0 & 0 & 0 & 0 & 1 & 0 & 0 \\
\hline 22 & 0 & 1 & 1 & 0 & 1 & 1 & 1 & 0 & 0 & 0 & 0 & 0 \\
\hline 23 & 0 & 1 & 1 & 0 & 1 & 1 & 0 & 1 & 0 & 0 & 0 & 0 \\
\hline 24 & 0 & 1 & 1 & 1 & 1 & 0 & 0 & 0 & 0 & 1 & 0 & 0 \\
\hline 25 & 0 & 0 & 0 & 1 & 0 & 0 & 0 & 0 & 0 & 0 & 0 & 0 \\
\hline 26 & 0 & 0 & 0 & 0 & 1 & 0 & 0 & 0 & 0 & 0 & 0 & 0 \\
\hline 27 & 0 & 0 & 0 & 0 & 1 & 1 & 0 & 0 & 0 & 0 & 0 & 0 \\
\hline 28 & 0 & 0 & 0 & 0 & 1 & 1 & 1 & 0 & 0 & 0 & 0 & 0 \\
\hline 29 & 0 & 0 & 0 & 0 & 1 & 1 & 0 & 1 & 0 & 0 & 0 & 0 \\
\hline 30 & 0 & 0 & 0 & 0 & 0 & 1 & 0 & 0 & 0 & 0 & 0 & 0 \\
\hline 31 & 0 & 0 & 0 & 0 & 0 & 1 & 1 & 0 & 0 & 0 & 0 & 0 \\
\hline 32 & 0 & 0 & 0 & 0 & 0 & 1 & 0 & 1 & 0 & 0 & 0 & 0 \\
\hline 33 & 0 & 0 & 0 & 0 & 0 & 0 & 0 & 1 & 0 & 0 & 0 & 0 \\
\hline 34 & 0 & 0 & 0 & 0 & 0 & 0 & 0 & 0 & 1 & 0 & 0 & 0 \\
\hline 35 & 0 & 0 & 0 & 0 & 0 & 0 & 0 & 0 & 0 & 0 & 1 & 0 \\
\hline 36 & 0 & 0 & 0 & 0 & 0 & 0 & 0 & 0 & 1 & 1 & 0 & 0 \\
\hline 37 & 0 & 0 & 0 & 0 & 0 & 0 & 0 & 0 & 0 & 0 & 1 & 1 \\
\hline 38 & 0 & 0 & 0 & 0 & 0 & 0 & 0 & 0 & 1 & 0 & 1 & 0 \\
\hline 39 & 0 & 0 & 0 & 0 & 0 & 0 & 0 & 0 & 0 & 0 & 0 & 1 \\
\hline 40 & 0 & 0 & 1 & 0 & 0 & 0 & 0 & 0 & 0 & 0 & 0 & 0 \\
\hline 41 & 0 & 0 & 0 & 0 & 0 & 0 & 0 & 0 & 0 & 1 & 0 & 0 \\
\hline
\end{tabular}

\section{Genetic Algorithm}

GA which produces the optimized values of weights and biases that are required to construct such network. The data used in the application were taken from the training set of the neural network.

\section{A. The Implemented GA}

The genetic algorithm begins by giving the weight and bias values of NFDS as initial population. However, the genetic algorithm can find the minimum even with a less than optimal choice for initial range. And it is used to find a good topology and parameters for a neural network. Using the sum square equation

$$
f(x)=\sum_{i=1}^{n}\left(\mathrm{x}_{\mathrm{i}} 2\right)
$$


as the fitness function, GA determines the fitness level of each topology and parameters. Using fitness values, the genetic algorithm would then evolve a new population for the network to try. After several generations, a population of several "good" structures with parameters evolves and fittest topology and parameters are used as the best construction of the neural network.

The genetic algorithm uses the individuals in the current generation to create the offspring that make up the next generation. Then, the algorithm creates a sequence of new populations. To create the new population, the algorithm scores each member of the current population by computing its fitness value.

\section{Result And Discussion}

The output response of the weight and the bias values after calculation is shown in Table 4, 5. This means that the constructed neural network is the best for the reactor accidents data. While the best structure is obtained from the Genetic Algorithm with the correspondence values of weights and biases, that are required to construct such network, are also calculated by the program.

\begin{tabular}{|c|c|c|c|c|c|}
\hline-3.0598 & -5.9107 & -7.6255 & 6.3460 & -9.1318 & -4.4427 \\
\hline-1.4349 & 1.0745 & -1.0691 & -2.1741 & -2.0210 & 0.5078 \\
\hline-8.7055 & -7.1797 & -1.1135 & 1.6681 & 3.4056 & 8.9190 \\
\hline 3.1601 & 1.2430 & -1.4179 & 0.2120 & -3.1159 & 0.4853 \\
\hline-2.4767 & -1.7021 & -0.9639 & -0.7843 & -3.4093 & -3.5982 \\
\hline-1.9175 & -2.4987 & -0.6356 & 2.1475 & -1.4463 & -1.2860 \\
\hline 0.0603 & -4.3219 & 3.9651 & 0.4637 & -4.2411 & -2.4960 \\
\hline 0.6917 & 0.2384 & 1.0230 & -1.1231 & 2.0335 & 0.8169 \\
\hline-4.6820 & -5.4202 & -1.5904 & -4.9911 & -3.5718 & -0.8605 \\
\hline 2.7301 & 1.3803 & 5.2333 & -2.7516 & -3.4596 & -0.3407 \\
\hline
\end{tabular}

Table 4: Layer 1 Weight Matrix $\{$ IW11 $\}$ 10x6

\begin{tabular}{|c|c|}
\hline$\{$ bi1 $\}$ & GA: $\{$ bi1 $\}$ \\
\hline 1.7215 & 4.9176 \\
\hline-2.0088 & 2.0049 \\
\hline-7.2876 & -0.1624 \\
\hline 1.4555 & -0.0898 \\
\hline 1.2405 & 0.4184 \\
\hline 2.0903 & -0.4712 \\
\hline 1.4614 & 0.9593 \\
\hline 0.4036 & -0.2859 \\
\hline 7.8119 & -0.3975 \\
\hline-3.2192 & 0.6909 \\
\hline
\end{tabular}

Table 5: Layer 1 Bias Matrix $\{$ bi1 $\}$

A. Comparison of NFDS and GA Outputs

\begin{tabular}{|c|c|}
\hline NFDS O/P FOR CASE 5 & GA UPDATED O/P FOR CASE 5 \\
\hline 0.1128 & 0.9997 \\
0.2488 & 0.9938 \\
0.0001 & 0.0000 \\
0.0001 & 0.0002 \\
0.0001 & 0.0000 \\
0.0090 & 0.0000 \\
0.0135 & 0.0361 \\
0.0001 & 0.0000 \\
0.0082 & 0.0444 \\
\hline
\end{tabular}

Table 6: NFDS output and GA output for case 5

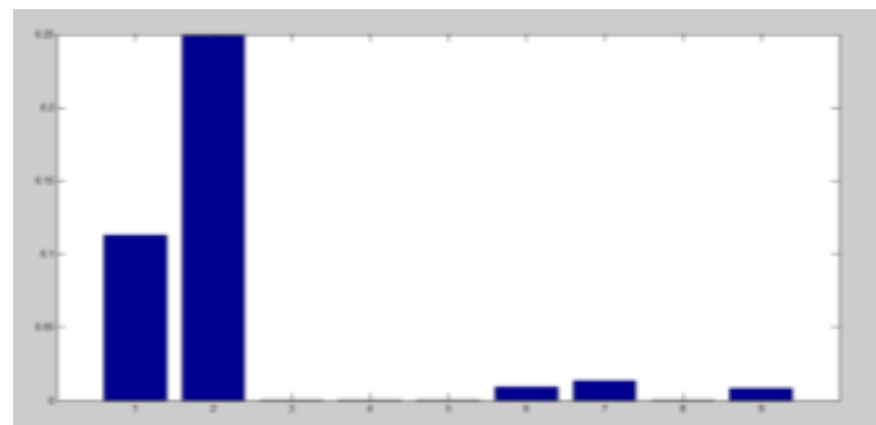

Fig. 5: output for NFDS case 5 


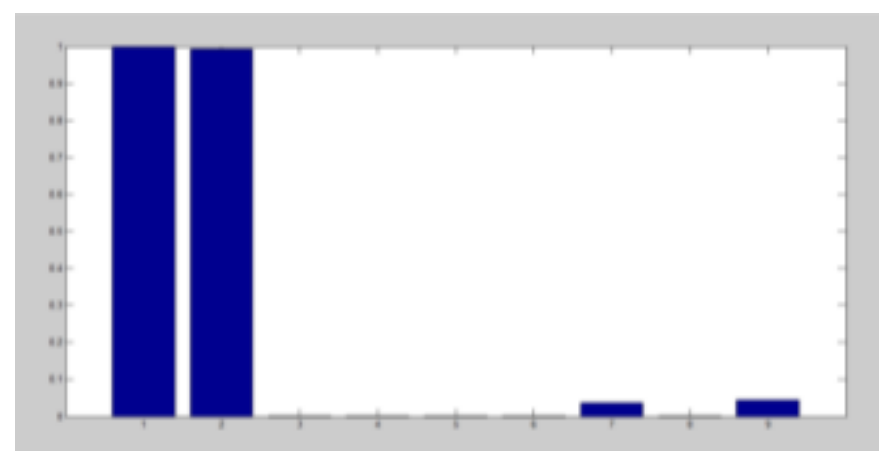

Fig. 6: output for GA case 5

\begin{tabular}{|c|c|}
\hline NFDS O/P FOR CASE 7 & GA UPDATED O/P FOR CASE 7 \\
\hline 0.9950 & 0.9997 \\
0.9563 & 0.9938 \\
0.0001 & 0.0001 \\
0.0002 & 0.0002 \\
0.0000 & 0.0000 \\
0.0004 & 0.0277 \\
0.0218 & 0.0361 \\
0.0000 & 0.0000 \\
0.0827 & 0.0829 \\
\hline
\end{tabular}

Table 7: NFDS output and GA output for case 7

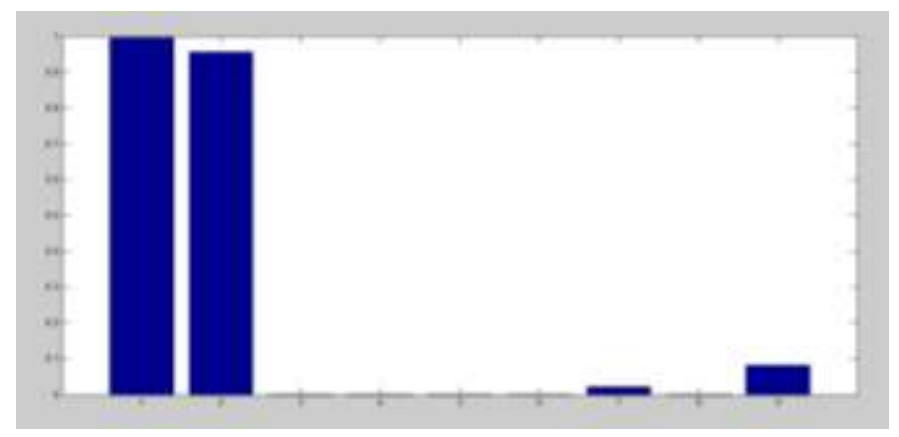

Fig. 7: output for NFDS case 7

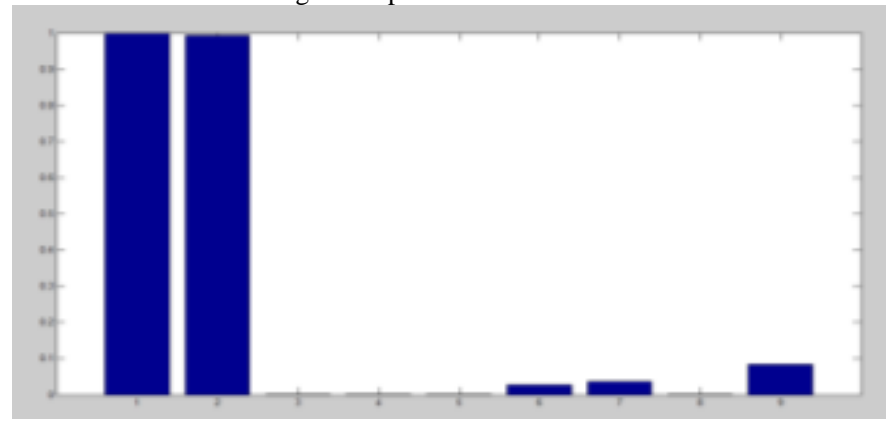

Fig. 8: output for GA case 7

\section{Conclusion}

In this paper a method was proposed for constructing an optimized Neurofuzzy diagnosis system by employing genetic algorithm the Neurofuzzy approach is almost appropriate for pattern recognition problems in environments where plant actual data are abundant and noisy. Moreover, the Neurofuzzy based systems can run very fast if hardware implementations are becoming available. This makes the systems, especially well suited for real-time applications such as alarm processing and fault diagnosis in NPR

In this method we implemented a genetic algorithm which can construct the high performance neural network structure for a given input nuclear reactors measured data, and the corresponding target accident. This method can be applied to any problem, where a data of inputs and outputs has the same form. In the reactor accidents diagnosis, the binary encoding becomes practical as the accidents patterns are in binary form. A 
comparison is conducted between a NFDS and GA. By applying the results obtained from genetic algorithms as simulating values the results showed much faster in testing the data, better convergence (smaller mean square error). This work demonstrated the method of automating and optimizing the design and finding weights and biases for a suggested construction of Neuro Fuzzy Diagnosis System by Genetic Algorithm in the domain of nuclear reactors.

\section{References}

[1] B. Lu, B.R. Upadhyaya, "Monitoring and fault diagnosis of the steam generator system of a nuclear power plant using data-driven modelling and residual space analysis", Annals of Nuclear Energy, Vol. 32, pp.897-912, 2005.

[2] A. Aboshosha, F. A. Mohamed, M. Elbardiny, N. El-Rabaie and A.Montasser, "Using Neural Networks in Fault Diagnosis of Nuclear Power Reactor", Proceedings of Fourth IEEE International conference, Electronics , Circuits, and systems ICECS 97, Cairo, Egypt, December 15-18, 1997.

[3] Keehoon Kim, Bartlett, E.B, "Nuclear power plant fault diagnosis using neural networks with error estimation by series association", IEEE Transactions on Nuclear Science, Vol.43, Issue 4, Aug. 1996, pp. 2373 - 2388

[4] Mustaf beyli, Elif Derya beyli, "Using recurrent neural networks for estimation of minor actinides' transmutation in a high power density fusion reactor", Expert Systems with Applications, Vol. 37, pp. 2742-2746, 2010.

[5] Kun Mo, Seung Jun Lee, Poong Hyun Seong, "A dynamic neural network aggregation model for transient diagnosis in nuclear power plants", Progress in Nuclear Energy, Vol.49, pp. 262-272, 2007.

[6] Enrico Zio, Piero Baraldi, Irina Crengut_a Popescu, "A fuzzy decision tree method for fault classification in the steam generator of a pressurized water reactor", Annals of Nuclear Energy 36, pp. 1159-1169, 2009.

[7] K. Zhao, B.R. Upadhyaya , "Adaptive fuzzy inference causal graph approach to fault detection and isolation of field devices in nuclear power plants", Progress in Nuclear Energy, Volume 46, Issues 3-4, Pages 226-240, 2005.

[8] E. Zio, G. Gola, "Neuro-fuzzy pattern classification for fault diagnosis in nuclear components", Annals of Nuclear Energy, 33, pp.415-426, 2006.

[9] L. S. Admuthe and S. D. Apte, "Neuro-Genetic Cost Optimization Model: Application of Textile Spinning Process", International Journal of Computer Theory and Engineering, 1793-8201, Vol. 1, No. 4, October2009.

[10] I. Kusco and Ch. Thornton, "Design of Artificial Neural Networks Using Genetic Algorithms: review and prospect", Cognilive and Cornpuling Sciences, University of Sussex, Brighton BN1 9QN, April 30, 1994.

[11] R. Hamdi, M. Bedda, "Arabic Speech Synthesis Using Optimized Neural Networks with Genetic Algorithms", Department of Electronic, Faculty of the Engineer, Annaba University, Algeria, Asian Journal of information Technology 5(7): 686-690, (C) Medwell Online, 2006.

[12] Mario D. Carelli , L. E. Conway, L. Oriani, "The design and safety features of the IRIS reactor", Nuclear Engineering and Design, Vol. 230, pp. 151-167, 2004.

[13] W.E. Cummins, M.M. Corletti, T.L. Schulz, "Westinghouse AP1000 Advanced Passive Plant", Proceedings of ICAPP '03 Cordoba, Spain, May 4-7, 2003. 